\title{
Broadband subwavelength sensitivity to symmetry defects of disordered slabs
}

\author{
Élie Chéron ${ }^{*}{ }^{*}$ Simon Félix, and Vincent Pagneux \\ Laboratoire d'Acoustique de l'Université du Mans (LAUM), UMR 6613, Institut d'Acoustique - Graduate School (IA-GS), \\ CNRS, Le Mans Université, Le Mans, France
}

(Received 13 February 2020; revised 13 September 2020; accepted 16 September 2020; published 1 October 2020)

\begin{abstract}
We observe a broadband and subwavelength sensitivity of the recently reported enhanced transmission through symmetric diffusive slabs [Chéron et al., Phys. Rev. Lett. 122, 125501 (2019)]. From a perfectly symmetric distribution of scatterers on both sides of an opaque barrier within a waveguide, a shift as small as $\lambda / 200$ significantly alters the multiple interferences giving rise to the transmission enhancement, and this subwavelength sensitivity appears on a very broad frequency range and without averaging. Analyses, from either full wave numerics or random matrix theory, show how the minimum shifting distance to suppress the transmission enhancement scales with the wavelength and the strength of the barrier.
\end{abstract}

DOI: 10.1103/PhysRevB.102.134201

A recent paper by the authors reported on a significant and broadband enhancement of the transmission through an opaque barrier when placed between symmetric diffusive disordered slabs [1]. This intriguing phenomenon is induced by multiple scattering interferences, which are inherent to the diffusive transport of waves, and which lead to well-known phenomena such as universal conductance fluctuations, enhanced back of forward scattering, or the Anderson localization [2-5]. Though the wavelength is naturally a characteristic length of such coherent effects, together with, typically, the mean-free path, subwavelength-sensitive behaviors may also occur [6,7].

In this article we show a broadband and deeply subwavelength sensitivity to the barrier position of the symmetryinduced transmission enhancement. Numerical simulations using a direct solution of the wave equation or the random matrix theory shows that a subwavelength shift of the barrier from the symmetry axis alters significantly the transmission enhancement. In addition, we observe a scaling of the distance requires one to reduce significantly the enhanced transmission regarding the strength of the barrier and the frequency.

To begin, let us first recall the basic result of Ref. [1]. In a quasi-one-dimensional waveguide supporting $N$ propagating modes, a thin opaque barrier with transmittance $N \tau$, $\tau \ll 1$, splits a $L$-length disorder slab in two halves. We assume a diffusive transport of waves through the slabs, hence an energy transmission described by the Ohmic conductance $g_{\mathrm{D}}=N \ell /(L+\ell)[8-10]$, which allows for a direct determination of the mean-free path, $\ell$. For nonsymmetrical slabs (ordinary disorder), their effect on the transmission simply "cumulates" with that of the barrier to give a more opaque overall system (see the black and red curves in Fig. 1). Here the transmission is measured by the Landauer conductance $g=\operatorname{Tr}\left(\mathrm{TT}^{\dagger}\right)$, with $\mathrm{T}$ the transmission matrix of the propagating modes [11]. If the spatial distribution of scatterers now obeys a left-right mirror symmetry with respect to the barrier

*elie.cheron@univ-lemans.fr (symmetric disorder), then induced coherent effects lead to a significant increase in conductance (dark blue curve). Though this effect is broadband and can be observed from a single realization of the slab disorder, one may wonder about its robustness to, e.g., symmetry breaking or losses [12], or when shifting the position of the barrier from the symmetry axis. The last case reveals an unexpectedly strong, subwavelength, sensitivity, as shown in Fig. 1 (light blue curve). While the barrier is shifted only by $\delta x=\lambda / 200$, the typical level of conductance is now less than one half of its original value. This effect, beside being deeply subwavelength, is at the same time very broadband, occurring over the whole frequency range. Note that, to avoid possible overlapping between the scatterers and the barrier while moving the latter, a small gap (with width $w \simeq 1.2 \lambda$ ) has been introduced between the two slabs, without incidence on the observed enhancement phenomenon. Figure 2 illustrates the effect on the wavefield of a small displacement of the barrier, showing, at the right, a much smaller average amplitude of the field. It is relevant to mention that Whitney et al. also report on such a deeply subwavelength sensitivity to the barrier position in the chaotic transport through symmetric double quantum dots [13].

Let us now look more precisely at how the conductance varies with the position of the barrier. For this we consider, at a given frequency, the two, asymmetric and symmetric, configurations shown in Fig. 1, and, from this starting point, we vary the shifting distance of the barrier position, $\delta x$, in the range $[-\lambda / 2, \lambda / 2]$; see Fig. 3(a). The same is also done at other values of the frequency $(N=100)$ and barrier transmittance ( $\tau=0.006$ ); see Fig. 3(b). First, the "ordinary disorder" configuration, which displays no symmetry, is insensitive to the barrier position within the gap: when varying $\delta x$, the conductance slightly fluctuates around a value that corresponds to a simple sum of series resistances, $\langle g\rangle \simeq\left(2 / g_{\mathrm{D}}+1 / N \tau\right)^{-1}$. This purely diffusive interpretation explains why the position of the barrier is indifferent. Note that, $\tau$ being very small $\left(\sim 10^{-3}\right)$, this mean value is close to the transmittance of the barrier alone, $N \tau$. The symmetric case shows, however, a much stronger dependence on the shifting distance $\delta x$, in 


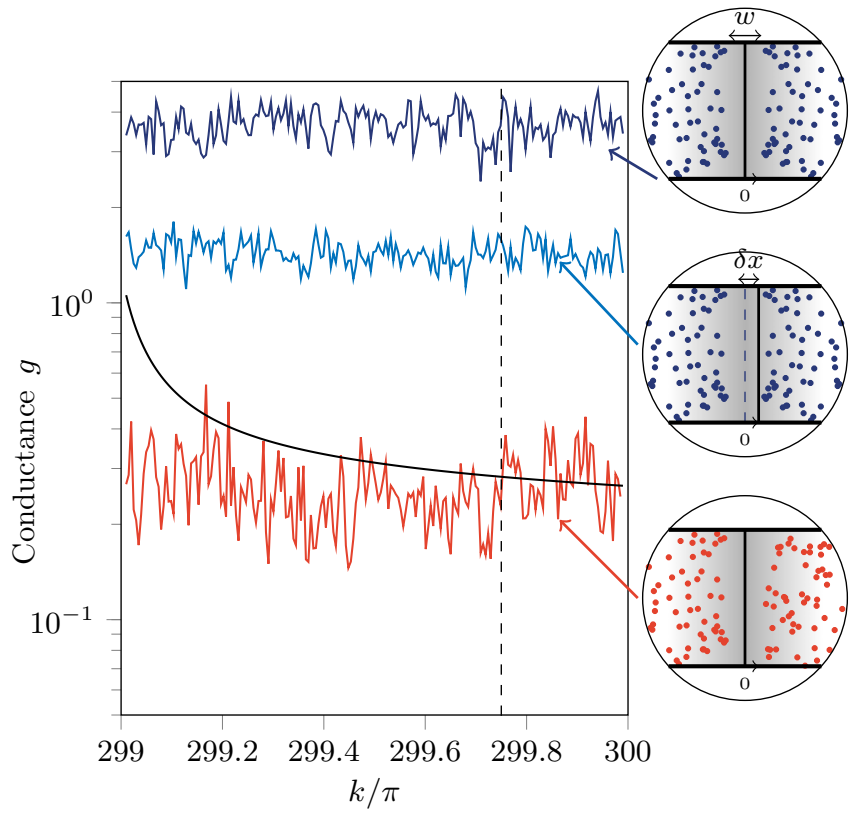

FIG. 1. Transmission through diffusive disordered slabs, separated, in the in-between gap, by a thin opaque barrier, in a waveguide of unit width. The conductance is plotted over the frequency range in which $N=300$ modes are propagating in the left and right homogeneous leads. Black: conductance $N \tau$ of the barrier alone (see Ref. [1] for more details). Red: the barrier separates two different slabs with length $L / 2=2.5$ and mean-free path $\ell=0.140$. Dark blue: same as red, but with the two slabs obeying a left-right mirror symmetry. Light blue: same symmetric disorder as the preceding configuration (dark blue) but with the barrier shifted by $\delta x \simeq \lambda / 200$ from the symmetry axis (to avoid an overlap between the barrier and the scatterers, a very thin gap is left between the slabs in the three configurations). In each configurations the conductance is obtained from a full wave numerical computation and comes from a single realization of the disorder (no averaging). The vertical dashed line indicates the configuration used to get Fig. 3.

particular near the position of perfect symmetry of the system, $\delta x=0$. When increasing $|\delta x|$ from zero, the averaged conductance drops so rapidly that, from $|\delta x| \sim \lambda / 50(\lambda / 30$ for $N=100$; see inset), it has reached the level of the asymmetric case, meaning that the symmetry-induced enhancement completely vanishes.

Furthermore, it keeps decreasing and stays below the "asymmetric" level until $\delta x$ is approximately one quarter of the wavelength. Work is in progress to further investigate this behavior, which, though robust, is not yet explained. For larger shifting distances, the asymmetric and the symmetric configurations give similar levels of conductance. No symmetry effect can be evidenced anymore.

To get further insight of the observed sensitivity, let us consider the conductance fluctuations in this specific slabbarrier-slab system. For the ordinary and the symmetrical configuration, the conductance can be written as the sum of its averaged value and a fluctuation: $g(\delta x)=\langle g(\delta x)\rangle+$ $\epsilon_{g}(\delta x)$. From the configuration shown in Fig. 3(a), we plot in Fig. 4 the normalized averaged fluctuation function $\langle\epsilon(\delta x)\rangle=$ $\left\langle\epsilon_{g}(\delta x) \epsilon_{g}(0)\right\rangle /\left\langle\epsilon_{g}^{2}(0)\right\rangle$, averaged over 50 realizations of
$-60$

0
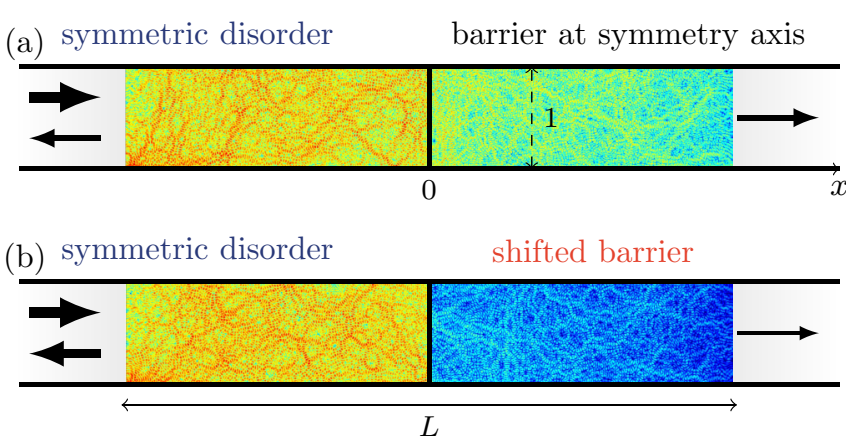

FIG. 2. Typical wavefield (magnitude, in log-scale) in two symmetric disordered slabs separated by a thin opaque barrier in the in-between gap, when impinged by incident unit fluxes on each of the $N$ propagating modes in the left lead. (a) The barrier lies on the symmetry axis, in the middle of the gap. (b) The barrier if shifted a distance $\delta x \simeq \lambda / 10 \simeq 0.004$ from the symmetry axis. In both cases the frequency is such that $N=50$, the total length of the slabs is $L=6$, and the mean-free path is $\ell=0.47$. The wavefield is obtained from full wave numerical computation, and the plots show its modulus in log scale.

disorder. The peak around $\delta x=0$ (see inset) gives the fluctuations of $g$ a characteristic subwavelength scale, $\lambda / 200$, regardless of the symmetry of the disorder. This scale is comparable to that observed in Fig. 3(a). Though this subwavelength property does not by itself rely on symmetry, it strongly influences the symmetry-induced conductance enhancement, making it subwavelength sensitive and, importantly, at any frequency and without averaging.

The two examples shown in Figs. 3(a) and 3(b), obtained at different values of both the frequency and the barrier transmittance, suggest that, beyond the common behaviors, the sensitivity, that is, typically, the width of the peak, depends on these parameters.

To investigate quantitatively this dependence, we start from the so-called optimal configuration, and we determine the displacement necessary to reduce the conductance by a factor of 2 . Given the mean-free path $\ell$ and barrier transmittance $\tau$, the optimal configuration is the symmetric system achieving the maximum conductance enhancement. It is obtained when the length of the slab is

$$
L_{\mathrm{opt}}=\ell\left[\sqrt{\left(\frac{1}{\tau}-1\right)\left(\frac{1}{\tau_{\mathrm{c}}}-1\right)}-\left(\frac{1}{\tau_{\mathrm{c}}}-1\right)\right],
$$

with $\tau_{\mathrm{c}} \simeq 0.4$ the threshold value above which no conductance enhancement is observed [1]. Then the averaged conductance reaches an optimal value $g_{\text {opt }}$. Note that this optimal configuration was defined and characterized in the absence of a gap between slabs, but we numerically checked that a narrow gap does not significantly modify these results. Now let us move the barrier from the symmetry axis of the system, until the averaged conductance is $g_{\text {opt }} / 2$. The corresponding shifting distance, noted $\delta l$, is plotted in Fig. 5 as a function of $\tau$, at various frequencies ( $N=50$ to 300$)$. 
(a)

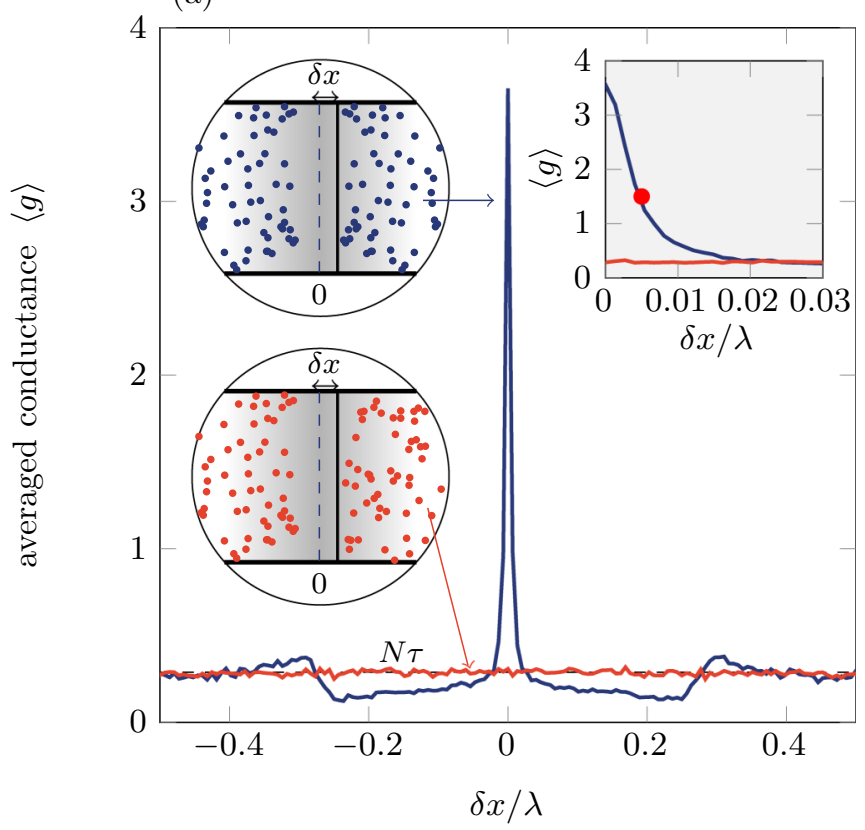

(b)

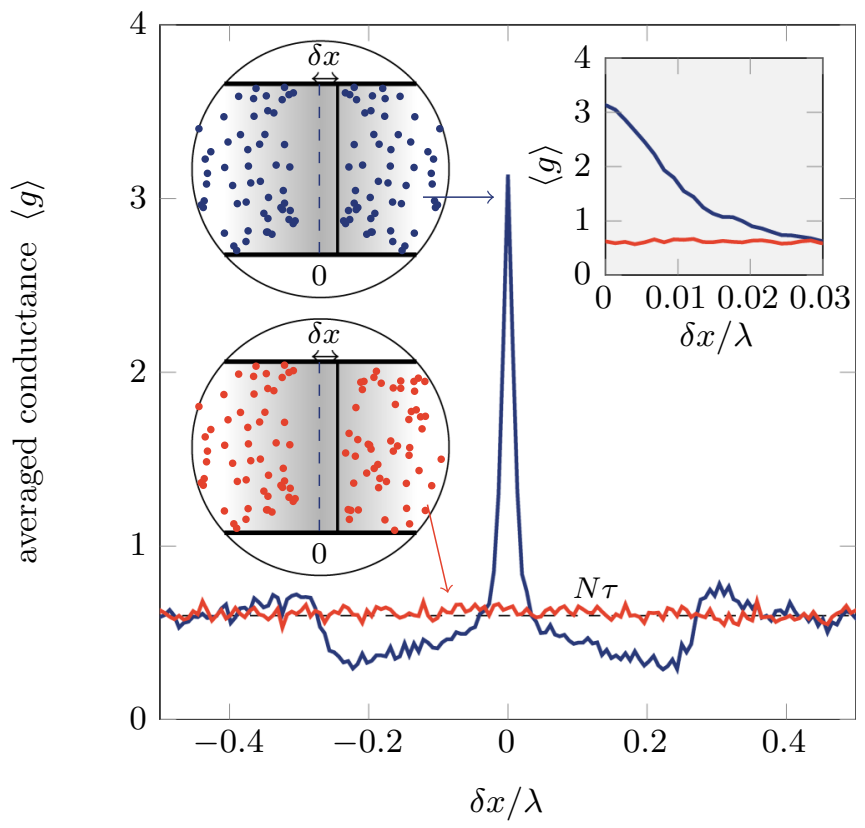

FIG. 3. Decrease in conductance when shifting the barrier a distance $\delta x$ from the symmetry axis, computed at two different frequencies, such that (a) $N=300$, (b) $N=100$. At each frequency, both the system with ordinary disorder (red) and the one with symmetric disorder (blue) are compared. In the abscissa, the shifting distance $\delta x$ is given in wavelength unit. Other parameters are (a) $L=$ $5, \ell=0.140, \tau=0.001$ and (b) $L=10, \ell=0.61, \tau=0.006$. Note that case (a) corresponds to the configuration shown in Fig. 1, at the frequency denoted by the red dot. The conductance in each case is obtained from full wave numerical computations and averaged over 50 realizations. The barrier transmittance, $N \tau$, is plotted for reference (black dashed line). Inset: zoom on the peak: $0 \leqslant \delta x / \lambda \leqslant 0.03$.

First, we observe that the sensitivity of the system, as measured by $\delta l$, increases with the opacity of the barrier

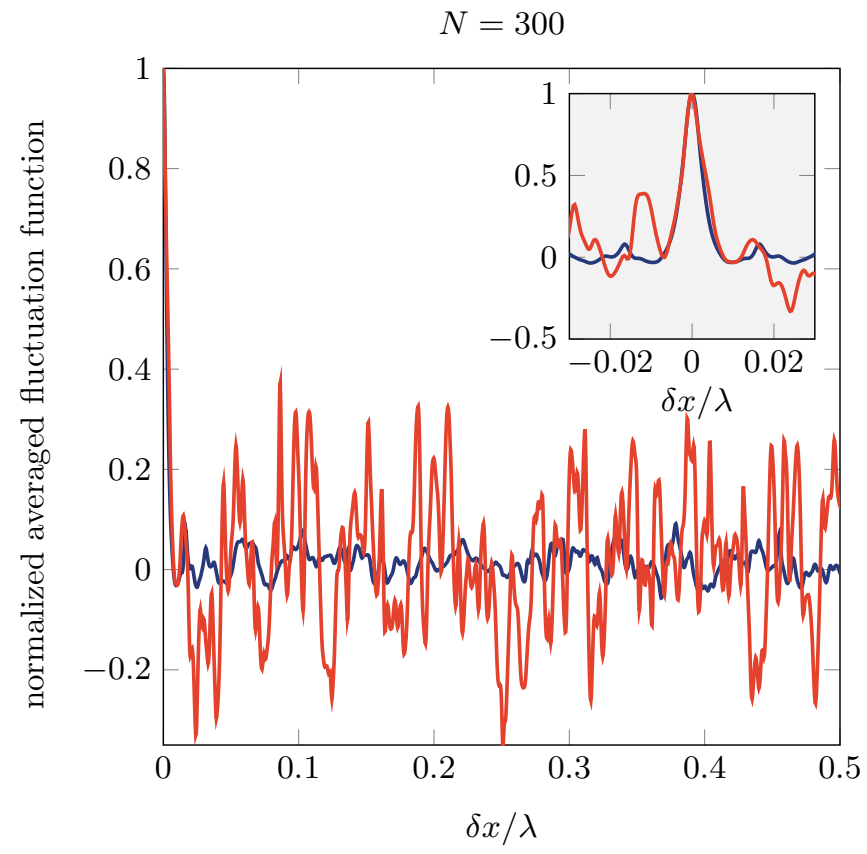

FIG. 4. Normalized averaged fluctuation as a function of the barrier shift $\delta x$ for a system with ordinary disorder (red) and with symmetric disorder (blue). The configuration is the same as in Fig. 3(a). Inset: zoom on the $-0.03 \leqslant \delta x / \lambda \leqslant 0.03$ region.

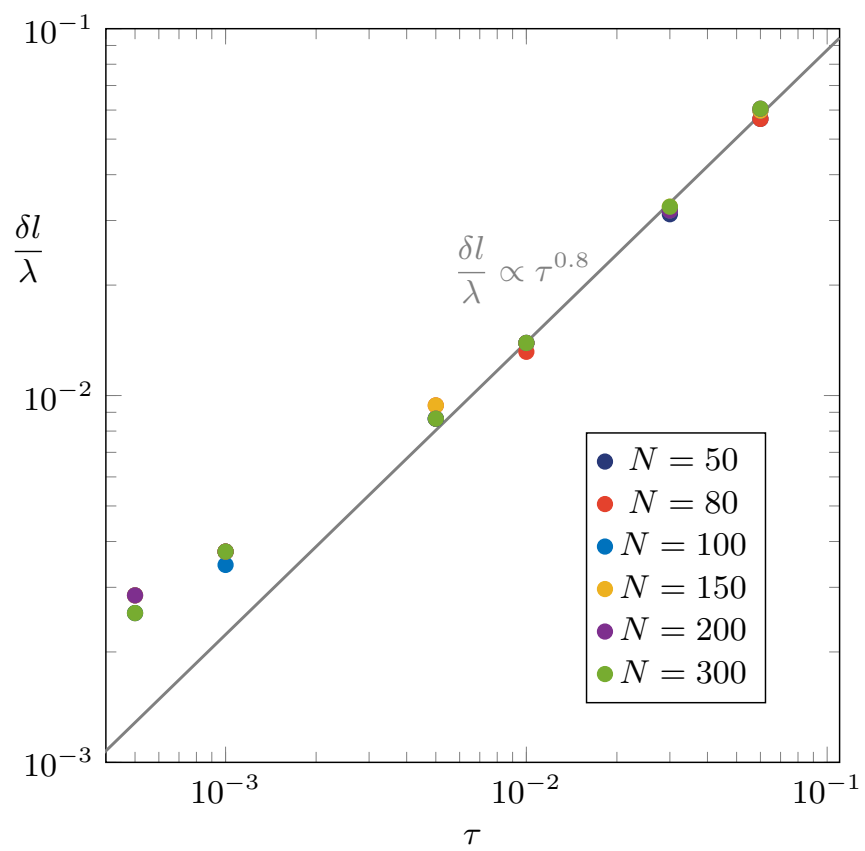

FIG. 5. Sensitivity of the symmetric slab-barrier-slab system with a shift of the position barrier from the symmetry axis, as a function of the barrier reduced transmittance, $\tau$, at various frequencies. Starting from an optimal configuration, as defined in Ref. [1], $\delta l$ is the minimal distance to lower the optimal conductance by a factor two. Computations are performed using random matrix theory [1,14]. Fit: $\delta l(\tau) / \lambda=a \tau^{b}$ with $a=0.5487 \pm 0.054$ and $b=$ $0.7968 \pm 0.0314$. 
(that is, $\delta l$ decreases with $\tau$ ). Also, this shifting distance $\delta l$, when expressed in wavelength unit, is almost independent of $N$. Then, a rough fit of the data gives $\delta l / \lambda$ simply scaling as $\tau^{0.8}$.

In conclusion, we have shown the broadband sensitivity of the transmission enhancement to a subwavelength shift of a barrier position between two symmetric diffusive slabs that can be observed without averaging. In addition, numerical simulations have shown how the minimum shifting distance needed to significantly lower the symmetry-induced transmission enhancement scales with the wavelength and the barrier

[1] É. Chéron, S. Félix, and V. Pagneux, Phys. Rev. Lett. 122, 125501 (2019).

[2] A. García-Martín and J. J. Sáenz, Wave Random Complex 15, 229 (2005).

[3] E. Akkermans and G. Montambaux, Mesoscopic Physics of Electrons and Photons (Cambridge University Press, Cambridge, 2007).

[4] T. Karpiuk, N. Cherroret, K. L. Lee, B. Grémaud, C. A. Müller, and C. Miniatura, Phys. Rev. Lett. 109, 190601 (2012).

[5] S. Rotter and S. Gigan, Rev. Mod. Phys. 89, 015005 (2017).

[6] F. Lemoult, M. Fink, and G. Lerosey, Phys. Rev. Lett. 107, 064301 (2011). transmittance. This high sensitivity to a symmetry defect, such as the sensitivity to losses [12], although it of course challenges the experimental observation of the symmetryinduced transmission enhancement, also opens the way to find nondestructive evaluation of defects in complex structured materials.

\section{ACKNOWLEDGMENT}

The authors would like to thank the referees for their helpful comments and suggestions.
[7] R. Pierrat, Ph.D. thesis, École Centrale de Paris, Paris, France (2007).

[8] O. N. Dorokhov, Solid State Commun. 51, 381 (1984).

[9] P. Mello, P. Pereyra, and N. Kumar, Ann. Phys. 181, 290 (1988).

[10] C. W. J. Beenakker, Rev. Mod. Phys. 69, 731 (1997).

[11] Y. Imry and R. Landauer, Rev. Mod. Phys. 71, S306 (1999).

[12] É. Chéron, S. Félix, and V. Pagneux, Sci. Rep. (to be published).

[13] R. S. Whitney, P. Marconcini, and M. Macucci, Phys. Rev. Lett. 102, 186802 (2009).

[14] N. Verrier, L. Depraeter, D. Felbacq, and M. Gross, Phys. Rev. B 93, 161114(R) (2016). 